\title{
BUILDING A SOUND AND FLEXIBLE EMERGENCY RESPONSE SYSTEM: HARD WON LESSONS IN DISASTER MANAGEMENT
}

\author{
George HANDY, John KIMBALL, and Jeffrey WINBOURNE
}

\begin{abstract}
Emergency management systems' failure to mitigate the devastation caused by hurricane Katrina imposed a number of unfortunate lessons from this disaster, lessons hard won and learned. These lessons are worth repeating for the benefit of the international emergency management community. Lessons this paper seeks to convey: the significance of organization and leadership, with communications as the critical link between them, to advance disaster responsiveness. Particular emphasis is placed on the role of communications, in its multiple modes, as it applies to disaster response management relevant to both Bulgaria and the Black Sea Basin/ Balkan region.
\end{abstract}

Keywords: Emergency Response System, Disaster Management, Hurricane Katrina, Leadership, Organization, Communications.

From our perspective, effective national response to disaster situations demands the following three core capabilities:

- Organization: Decentralized action and reaction bolstered by intense centralized operational support, organization, and policy direction, involving cooperation between civil and military government organizations, the private sector, and neighboring countries;

- Leadership: Leadership skill at all levels of the response to organization to motivate responders and direct cooperative action at all levels;

- Communications: Effective and timely sharing of information with emphasis on how, and how much information is acquired, how much and how frequently that information is shared, and how it is protected. 


\section{Unifying Theme}

Effective use of organization and leadership requires timely, accurate, and fully functional communications. Local response units must be able to communicate their situation, and paint an accurate picture of the incident to their commander and upward, or to operational support and senior leadership. Communications may range from "situation stable" status reports, to requests for a wide variety of assistance while indicating its urgency. The aftermath of hurricane Katrina suggests much needed scrutiny of the communication systems between federal, state, and local leadership.

\section{Organization}

Emergency management entities plan, prepare, and respond to a variety of crisis situations. Emergencies might range from manmade incidents, such as a chemicalbiological threat to the New York City subway system, to that of a hurricane the size and force of Hurricane Katrina. Organization allows resources to anticipate and effectively manage disaster response, minimizing loss of life and property. Ability to implement pre-designed plans plays a key organizational role in effective emergency responsiveness. However, the ability to communicate with all assigned organizational units, and to deploy assigned resources in a timely manner, is critical to the successful organization of any emergency situation.

Example: A suspect agent is discovered, and appears to be chemical-biological in nature. First responders associated with local jurisdictions request more sophisticated testing and analysis of the suspect agent. What constitutes communications procedure? Several actions occur simultaneously: The on-site first responders take appropriate action based on local protocols and their knowledge of the situation. Requests for advanced analytical resources are simultaneously communicated up the chains of command. Needed resources are drawn from national, interagency, and/or private sector resources, and regional and international entities. The level of resources required dictates the entity appropriate to the task.

A natural disaster, such as a flood or an earthquake, exacts similar responsiveness. On-scene first responders recognize the need for advanced search and rescue capabilities, as well as extensive and immediate evacuation and transportation assets. The support for these tactical requests can be provided at all levels of national response. Neighboring jurisdictions may support evacuation and search and rescue at their level of capability. Assistance from neighboring countries and international search and rescue organizations may be requested concurrently at the appropriate national senior leadership level. 
Hurricane Katrina impacted a geographic area approximately the size of Great Britain. Over 100 jurisdictions from local, state, and federal levels responded. Hundreds of non-government organizations also engaged, including the International Association of Fire Fighters and the National Organization for the Disabled, legitimate and valuable provisions of disaster response. Katrina's response requirements were obscured by the complex nature of lost assets, including fire protection, emergency medical services, and law enforcement. Entire infrastructure systems were obliterated, including those that provided food, water, housing and energy, as well as provisions for special needs populations. Countless industrial facilities in the region were almost completely destroyed. The national energy infrastructure was adversely impacted and numerous, major chemical spills migrated from the facilities, greatly adding to the local flood damage.

Without rapid and accurate communication systems, situations such as those described above become breeding grounds for rumor, panic, confusion, needless human suffering, excessive property loss, and probable loss of life. Such outcomes are preventable with the use of modern communication systems, comprehensive planning efforts, as well as aggressive training and exercise programs.

\section{Leadership}

Leadership guides and motivates responders to the extent that it has sufficient knowledge of the situation. Knowledge and preparation bolster responder confidence. The leader armed with critical and accurate on-site details performs based on pre-defined action plans, and instills response forces with confidence and unity. Such leadership provides motivational messages across geographic and political borders.

Mayor Giuliani's response following the September 11, 2001 terrorist attack in New York City illustrates this point. During the aftermath of the attack, the mayor gave direction to city resources responding to the attack, to the people of New York City, and to the nation. His ability to communicate with both general population and first responders was a critical element in providing leadership, rallying first responders to manage the tasks at hand, and addressing individual and societal feelings of grief and rage. As a leader, he understood his responsibility for managing local and national response to the attack. Several years prior to the attack, Mayor Giuliani advocated establishing the City's Office of Emergency Management. He recognized the role of organization, coupled with a sound communications infrastructure, as a critical tool for effectively managing emergency situations.

Another critical leadership concern involves effectively balancing competing demands for resources. The following illustrates the impact of pre-disaster planning at a national level: According to a US Government Accountability Office (GAO) report, 
more than $75 \%$ of next year's Department of Homeland Security's preparedness grants convert to state and local readiness for anti-terrorism resources. The leadership of the National Emergency Management Association identified the problem. It warned DHS leadership that the change, including more proposed reductions of the Federal Emergency Management Agency (FEMA) role, weakened readiness for disaster response. Shifting resource allocation inappropriately to one DHS mission at the expense of another downplayed the role of emergency and disaster planning and response, and its overarching need to improve interoperable communications between emergency responders at all levels.

The conclusion and challenge: A disaster scenario requires leadership responsiveness to the dynamic, real-time demands of the situation. It must possess a foundation of solid preparation, effective organization, and comprehensive communications capabilities on which to rely. Additionally, prior to a disaster, leadership must provide strategic policy direction required for funding and support of preparedness measures.

\section{Communications}

Communications observably enables the bond between leadership and organization. When first responder units cannot communicate with the command structure or with each other, they became isolated and ineffective. If organization cannot communicate with both leadership and on-site units, effectiveness of units becomes marginalized.

Communication inadequacies between diverse units of first responders were patently critical to the Katrina response, especially with failure of existing electrical and telecommunications infrastructure. Planning for future events such as Katrina requires rethinking current approaches to communications technology. Use of independent communications platforms such as those used by the military becomes critical to future planning. Satellite communications, which received much attention after September 11, require consideration for use in all-hazards scenarios. The design and location of emergency communications centers and operational command centers also requires addressing. The new design must proceed from consequences: the over 50 centers that were inoperable for a time, or completely destroyed. Essential to disaster planning and communications is a high degree of reliability and redundancy for the response center buildings, their base-building systems, as well as the voice, data and video systems used by center personnel.

The impact on communications of both Katrina and September $11^{\text {th }}$ emphasizes our dependence on existing infrastructures. The magnitude of Katrina's destruction prevented first responder units from communicating for reasons including equipment and frequency incompatibility, with all telephone system types effectively under water and out of service. September $11^{\text {th }}$ first responders experienced similar, well docu- 
mented, communications problems. The city's electrical grid was severely impacted, limiting power to radio and telephone systems that primarily, and in many cases, depended on it exclusively for powering these systems.

Another significant communications issue to consider involves discerning how much information should be passed through the chain of command's varying levels. Information overload becomes a common problem at emergency incidents of all types. Technology assists in classifying the type of information and its transmission. However, the information must be acquired, analyzed, and transmitted from the incident to each succeeding level in the response continuum. This concept derives from a combination of communications technical expertise, and the knowledge, experience, and training level of those analyzing the information. Procedures must also be developed in this regard that guide personnel, and can be practiced in exercises and planning events for all-hazards scenarios.

First responders must be able to filter out what is immediately important to develop objectives, and to identify the resources needed to accomplish those objectives. This activity is repeated at each step up the chain of command. The frequency of information transmission and updates can be managed by adopting the Incident Action Plan (IAP) method. The IAP clarifies task level actions, tactical goals, and strategic objectives at each level or organization on a time-phased approach based on the priorities of the incident. The IAP is a tested and proven method for focusing the information flow, and enabling response managers at all levels to make better informed decisions based on accurate information. Not only is the IAP a format designed to assist in the decision-making process, it is also an excellent method of communicating the decisions, and evaluating the effectiveness of the decisions. Responders at each level should be empowered by procedures and activated by accurate information in order to perform immediate critical actions, and integrate those actions into the longer-term Incident Action Plan. Responder and other personnel should not be burdened with information they do not need.

One of the immediate lessons learned from the effects of Hurricane Katrina concerns leadership, and its ability to capture and accurately interpret information. While much of the discussion surrounding real and perceived failures of governmental disaster response is skewed by natural human emotions, and fueled by the extent of the disaster and the personal hardships incurred, analytical conclusions can be drawn. Leadership at all levels lacked an accurate picture of the size, scope, potential, and extent of the storm and its resultant impact. While responders at all levels performed to the limits of human endurance and beyond, deficient information caused by poor or non-existent communications resulted in less-than-informed decisions and planning by leaders at every level. 
Today's fast developing emergency situations, with increasingly capable communications technology, establishes information sharing systems as the cornerstone for emergency response. These systems are expected to rapidly and unerringly receive current disaster descriptions, assign resources, and modify standing policies and orders when appropriate. Additionally, leadership, through the emergency communications systems, is expected to quickly shift resources as the initial crises are mitigated, and more serious requirements identified.

\section{Bulgaria's Emphasis on Information Sharing}

Bulgarian planning for emergency management system modernization reflects each of these three capabilities, leadership, organization, and communication, with a priority on improving the latter. The present system limits efficient transmission of information between first responder agencies, including police, fire, and emergency medical. As we understand it, the Bulgarian Civil Protection Agency (CPA) is establishing an all-hazard response capability to address recurring natural hazards such as earthquakes and floods, as well as assigning appropriate emphasis to threats such as terrorism and hazardous waste. Additionally, CPA currently addresses technical infrastructure challenges, ranging from emergency communications capabilities to new communications requirements associated with integration into NATO and the European Union (EU). Establishing national communications architecture for civil protection information sharing, including interoperable systems supporting all-hazards operations, presents a clear challenge.

A recently published document of the US Department of Homeland Security Office of the Inspector General, “OIG-05036” detailed some critical conclusions concerning information technology applicable to Bulgaria and the Balkan/ Black Sea region. The report concerned assessing the strengths and weaknesses of the DHS Emergency Preparedness and Response Directorate to support incident response and recovery operations. The report highlights the DHS response to the previously unprecedented hurricane season of 2004 in which the east coast of the US was stuck by four hurricanes and one tropical storm in under three months. The report confirmed that the incident management system and the information technology needed to support such repeated responses was adequate to meet most of the challenges presented by these storms. However, the report indicates that the system was stressed nearly to the breaking point.

The conclusions and recommendations of this report were unfortunately validated by hurricanes Katrina and Rita in 2005. These include the following points:

- Modernization of outdated and dysfunctional legacy IT systems, including development and construction, which must support the national and regional 
strategic goals and operational objectives of disaster planning and response. The IT strategic plan must reflect the national strategic plan, and IT systems must integrate to effectively support information exchange during response and recovery operations.

- Long-term solutions are far preferable to short-term fixes, especially when the existing infrastructure is outdated and based on similarly outdated organizational processes.

- Updating must include adequate requirements definitions, alternatives analyses, and sufficient tests prior to deployment

\section{Reliable and Flexible Solutions}

The manner in which the command, control and communications systems connect the first responder with city, province, and national leaders must improve logistical support, timely shifts in priorities, and deployment of first responder resources for an effective emergency response.

The following are considerations for how the solutions can be achieved:

- Leverage technology to insure agility and reliability in response to changing conditions, and planning for all-hazards conditions.

- Ensure effective decision-making through informed leadership and organization.

- Test leadership, systems, and organization through routine, "real-life” planning exercises.

\section{Concluding Thoughts}

By examining past emergencies and the current planning of major U.S. cities, states, and the national government, two measures of success stand out: establishing an effective single point of leadership for emergency response, and correctly managing first tasks in an emergency. Both leadership and first tasks depend upon effective information sharing through modern command, control, and communications systems. Determining the "first task," or priory tasks, depends upon trained, experienced leaders familiar with the disaster scenario they are facing.

Without reliable, redundant, and competent communications to acquire and transmit useful information, even the finest leaders and response organizations face probable failure. Conversely, in the absence of trained leaders and organizations, the most robust communication system composed of state-of-the-art technology becomes a waste of national resources. 
A national emergency management program requires leadership that provides strong strategic direction. Critical to strategy is its link to an organizational development plan of training, drills, and exercises. The national organization should be complemented by local and regional organizations that utilize the same operating protocols and procedures customized to local conditions. Linking this constellation of organizations and resources requires resilient, multi-mode communications systems that can withstand the results of a variety of natural and manmade disaster scenarios. Failure to integrate information technology systems with organization, planning, procedures, and training indicates failure of government in its duty to protect its citizens. Without this approach, the hard won lessons taught by both Katrina and September $11^{\text {th }}$ will fail to have been fully learned, and the consequences doomed to become recurring themes at future disaster scenes.

GEORGE HANDY works for the Center for Strategic and International Studies (CSIS), concentrating for over 14 years on Eastern and Central European issues. He also serves as a Senior Advisor to Winbourne \& Costas, Inc. regarding Eastern and Central European regional issues and emergency management.

JOHN KIMBALL is the Director of Emergency Management Services with Winbourne \& Costas, Inc. He has over 20 years of emergency management experience working with the US Federal Emergency Management Agency (FEMA), and the US National Fire Academy. He has worked in many countries on emergency management issues, including: Bulgaria, United Arab Emirates, Macedonia, Greece, Turkey, Saudi Arabia, Singapore, and others.

JEFFREY WINBOURNE is the President and a founder of Winbourne \& Costas., Inc. He is a recognized expert on emergency communications. He has provided expert advice to US cities including Washington, DC, New York City, Philadelphia, and Miami.

Winbourne \& Costas, Inc. is a US-based firm that specializes in emergency management and communications consulting services. 